\title{
Material Innovation of Animation Modeling Design Based on Visual Symbol Theory
}

\author{
Xuerui Cao \\ Xijing University, Xi'an, Shaanxi 710000, China \\ Correspondence should be addressed to Xuerui Cao; 20140063@xijing.edu.cn
}

Received 3 January 2022; Revised 20 January 2022; Accepted 21 January 2022; Published 18 February 2022

Academic Editor: Xin Ning

Copyright ( 12022 Xuerui Cao. This is an open access article distributed under the Creative Commons Attribution License, which permits unrestricted use, distribution, and reproduction in any medium, provided the original work is properly cited.

Character modeling is an important link in animation design, and it is one of the keys to determining whether an animation is successful or not, as well as determining the value of animation and related industries. This paper examines the role of material innovation in the development of animation art, as well as the impact of various materials on animation production technology and design style, using the visual symbol theory as a guide. GA (genetic algorithm) is used in the comic design process to improve the fidelity and efficiency of the artwork. GA performs data training of multiple components; selection and crossover operations are carried out continuously according to the fitness threshold; and the particle swarm optimization strategy is added. This algorithm increases the rate of design reuse and efficiency, as well as the ability to determine the best component combination scheme.

\section{Introduction}

With the rapid development of electronic media technology, animation in the new century has become the most familiar art form in world pop culture [1]. The animation method has stepped into the "computer-made era" from the "handmade era" in the last century. The animation materials have undergone fundamental changes. Symbolized thinking and behavior are the most representative features of human life, and all the development of human culture depends on these conditions [2]. The design idea of formal beauty is difficult to deal with all kinds of complicated information. The symbol is also the material means of human thoughts and feelings. Human beings use symbols to communicate and exchange knowledge [3, 4]. Symbol connects our culture, life, politics, and economy in series, and its combination, transformation, and regeneration guide our thinking orderly.

In the animation design work, make full use of different elements, combine them, and reconstruct them, so as to form different images [5]. Therefore, animation modeling design combines painting skills, film editing skills, language art, and so on and shows the art form in a more systematic way. The costumes and props of cartoon characters are important factors to show the characteristics of cartoon characters. How to vividly show cartoon characters in front of the audience puts forward higher requirements for designers. There are many kinds of fabrics for clothing props. Because of different characteristics, different fabrics have certain differences in technology and modeling $[6,7]$. From the physical attributes on the surface of the material to the psychological attributes inside the material, the unspeakable emotional semantics of the material are symbolized so that the artistic language contained in the comprehensive material can be deeply explored [8]. From the perspective of information dissemination, pay attention to the degree of correspondence between the form and meaning of visual symbols and how to effectively convey the information it carries. From the cultural point of view, study the relationship between the fusion or conflict of visual symbols and the era in which they live. How to construct the form of visual symbols succinctly, intuitively, and aesthetically will become the main content of the research on the direction of visual communication [9].

Designers must fully comprehend the design intent in the design drawings and select the most appropriate materials for assembly and processing in order to achieve the 
animation design effect. It is more effective to combine design work with animation design. Symbols and objects are relative, not absolute, and they can be transformed dynamically or with other things, resulting in a complex hierarchical and structural relationship between symbols and objects that is difficult to separate [10]. However, as people's visual sensory requirements improve, so do their material requirements, and the original materials can no longer meet people's visual aesthetic requirements. As a result, new technical materials emerged.

\section{Related Work}

The graphic expression composed of visual symbols can be indirectly understood as the interpretation of the visual characteristics of the graphic form. If the message conveyed by visual symbols cannot highly restore the existing cultural value, it will greatly reduce the connotation understanding value of animation works, thus affecting the recognition degree of the whole animation. As one of the most important communication carriers, the visual symbol is the key part of the core function of the symbol, which is responsible for the mission of spreading the cultural value behind the whole animation works [11]. Reference [12] holds that every symbol can be divided into symbols, such as words or designs on paper. Reference [13] holds that the meaning represented by symbols is determined according to the overall social and cultural system, and symbols themselves have no implied meaning. Reference [14] established the category of semiotics on the logic of the relationship between thinking and judgment. Symbols are defined in three functional senses, namely, identification, evaluation, and instruction. Reference [15] holds that human beings are symbolic animals, and all human spiritual culture is the product of symbolic activities. The essence of man is that he can use symbols to create culture. Reference [16] holds that language is only a subsystem of the whole symbol system, and only from the big symbol system can we truly gain insight into its inner mystery. Reference [17] holds that the process of visual symbols conveying information is the process of classifying, filtering, simplifying, and spreading all kinds of information in social life. As an intermediary of information dissemination, it not only can make the visual information hierarchical and achieve the effect of instantaneous information transmission but also can enable the audience to receive accurate information.

Many researchers, both at home and abroad, have worked tirelessly to gain a better understanding of animation culture and promote its development. Although it is more stereoscopic, the characters are more vivid, and the limitation of two-dimensional character action design is also broken through in Reference [18], there are some flaws such as high picture saturation and stiff color change. The development direction and modern elements of original animation illustrations are investigated and analyzed in reference [19]. However, no studies on the use of comprehensive materials in animation illustrations have been conducted. Reference [20] has provided a thorough examination of the meaning and symbolism of symbols, which has aided in the study of this subject. The artistic language and artistic charm of materials are elaborated in literature [21] from two major aspects: an artistic expression of materials and technological expression of materials, which is also the general direction of material research in this paper, and the emotional semantic symbols contained in materials are extracted and studied. Based on design psychology, literature [22] has conducted multidimensional analysis and product experience research, which has aided in the development of my research methods. Reference [23] provides a theoretical foundation for me to study comprehensive material emotions by delving into the relationship and principle between visual perception and art.

The globalization of the world economy is driving cultural exchanges around the world, and the design of visual symbols will promote international information dissemination from beginning to end. Because of this, it has become a common form of information transmission in the world, and some visual graphic symbols have already reached the international unified standard.

\section{Research Method}

3.1. Material Innovation in Animation Design. Animation modeling design can be said to be the core component of animation creation. It not only has extremely high artistic aesthetic value but also has unlimited commercial value and promotion space. In addition to its unique shape, the successful animated character modeling also has distinctive typical character and personality. The differences across races and cultures are accepted by the whole world, and the spiritual connotation injected into the character by the creator also spreads to the whole world. These animation shapes are representative animation characters in various countries. Case studies will be conducted on some of these animated characters, mainly analyzing and interpreting the character shapes from the aspects of their modeling methods, brand building, and derivative development and design.

The creative behavior of visual symbols is subject to cultural constraints, and there is no visual symbol without cultural constraints, and the visual effect eventually formed will inevitably reflect culture. The first step of creating behavior is to understand the social and cultural background, including functional requirements, target groups, customs and habits, color preferences, and so on. Only with this cultural information can creation be carried on. No matter whether they belong to any social stratum, they all stand in the background of this era, so the created results will inevitably reflect the cultural characteristics of the era.

The symbol is the creation of human beings, and its formation was born in artificial meaning. The process of implementing the meaning function is called the symbolic process, and the creation of symbols by human beings is realized in one process. All symbols are created through the symbolic process of illustration. The process and meaning of symbols are shown in Figure 1.

It should be noted that visual culture poses a challenge to both the traditional visual culture system centered on visual 


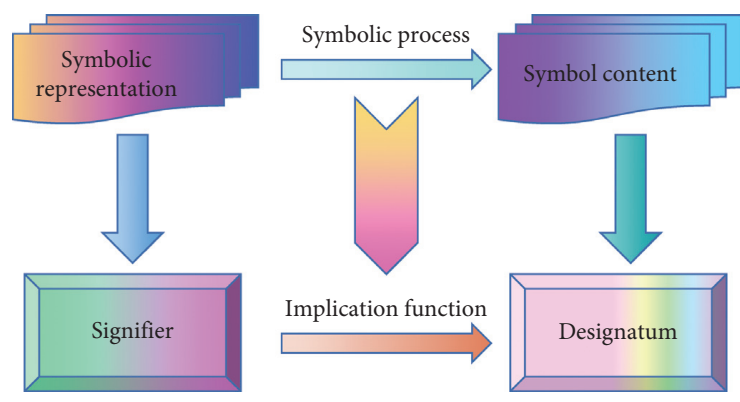

FIGURE 1: The process and implication of symbols.

symbols and the traditional language culture system centered on language symbols, causing modern culture to shift from rationalism to perceptual form centered on images. It is important to note that the evolution of visual culture and the evolution of language and culture are unrelated. Visual symbols will not take the place of language symbols, and visual culture will not take the place of language and culture. Images are unquestionably more appealing than words. Cartoons, movies, TV dramas, computer games, and other forms of entertainment are popular among not only children but also college students and ordinary adults due to their readability. Multimedia instruction has supplanted singlelanguage instruction, and television and the Internet have surpassed newspapers in popularity.

The practical functions of form symbols include the expression function of form symbols to designers' intentions, the significant function of form symbols to product cultural values, and the cognitive function of users to product cultural values through form symbols. In the process of conveying cultural values, the significant function is the prerequisite for the realization of expressive function and cognitive function. Figure 2 shows the practical functions of morphological symbols.

The appearance of new technical materials brings better display power to the animation character modeling design, which can make the modeling design change from static to dynamic. Dynamic character modeling forms are not the mainstream at present. New technical materials can change their shape, color, or pattern layout when triggered by environmental or electrical signals. The corresponding materials can be single materials, composite materials, or composite materials equipped with signal receiving and transmitting devices to realize control.

Currently, electric and magnetic signals can be used to activate electromagnetic structures in animation modeling design, allowing material types to be effectively changed. The magnet, which can effectively complete the application and application of current to ensure its magnetism, thus effectively improving the application effect of materials, is the most basic of electromagnetic materials. This type of material property change is instantaneous, allowing for effective short-term morphological change and complete control over the change effect. With the continued development of $4 \mathrm{D}$, $5 \mathrm{D}$, and other technologies, it is necessary to combine scientific and technological elements with modeling design concepts, effectively form a good macro-treatment scheme, ensure that the integrity of the animation modeling design process can be truly improved, and also provide guarantee for the integration of design elements in order to improve the effect of animation modeling design. After the displacement transmission is realized, different structural forms can be processed, ensuring that the basic operation can be made by bending screws or iron wires, and the data and movements can be effectively transmitted by pulling processing, ensuring the fluency of the animation design process and laying the foundation for the optimization of the overall movement structure's coordination degree.

Self-changing mode realized by inductive element and then combined with electric structure is one of the mainstream modes in shape change. The so-called electric structure refers to all kinds of structures that can be controlled by electric signals to generate macroscopic mechanical displacement or rotation. Transmission can be carried out by pulling with screws or iron wires, or by hydraulic or pneumatic transmission. For example, to make a design that can synchronously change shape at six positions, only one hydraulic pump is needed, and the hydraulic pressure is conducted to six positions through the hydraulic pump with a very thin liquid conduit so that the control operation of six positions can be conveniently realized, and no more auxiliary structures are needed.

3.2. Animation Design Optimization. In the process of animation design, as the carrier of artworks, materials have both natural and social attributes. The invariability of nature will restrict artists' creation and directly affect the formation of artistic skills and styles. The social attribute of materials is that artists can express their artistic ideas through the choice of materials. Therefore, materials have the function of carrying artistic works and interpreting artistic ideas.

In artistic creation, the richness, infinity, and regional characteristics of materials provide infinite inspiration for our artistic creation. The application of materials not only involves the problem of animation production technology but also directly affects the formation of animation art style. The application of new materials in animation promotes the emergence of new artistic language; On the other hand, the characteristics of materials directly affect the change of character's motion mode in animation production, which makes the animation picture from static to dynamic form a unique artistic style.

In animation production, animation modeling often needs to constantly change posture. In order to facilitate motion control, the creator needs to consider the character modeling and the characteristics of clay to design a strong and durable skeleton. At the same time, animators must also master the material surface treatment and finishing technology and process. Therefore, the level of animation production technology will directly affect the final effect of animation.

The main purpose of parallel processing is to speed up processing and shorten processing time. Parallel operation is an important way to solve the problem that numerical operation with a large amount of data takes too long. 


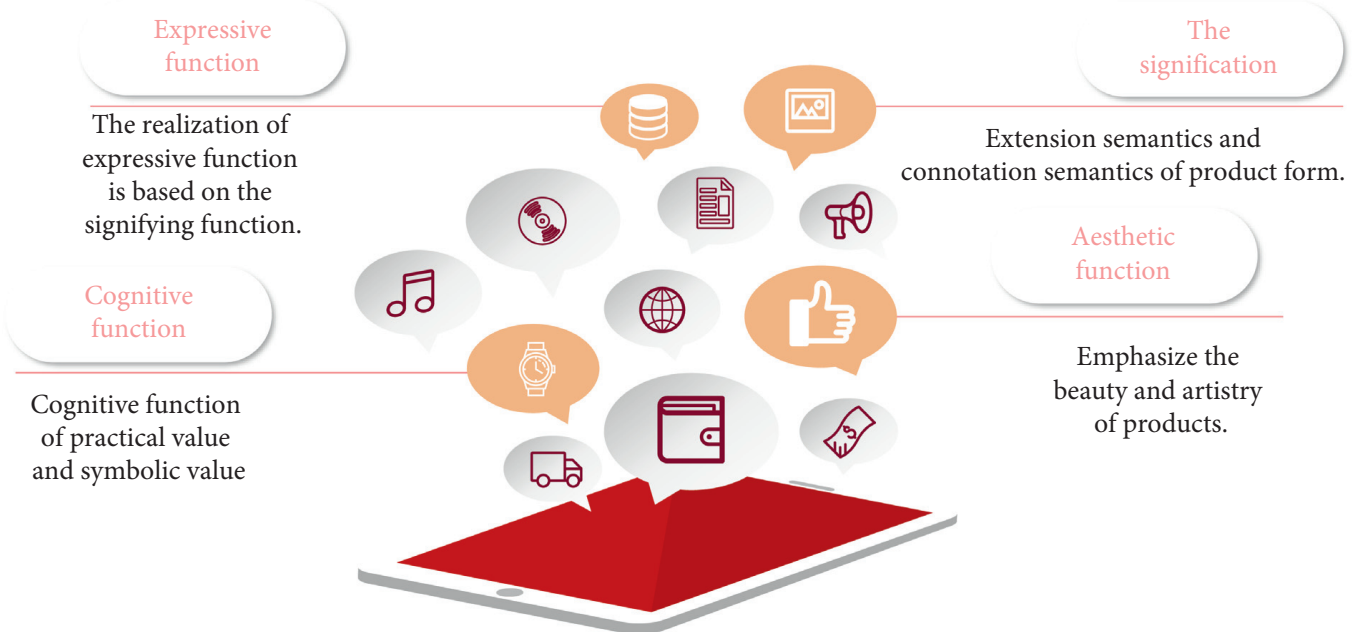

FIgURe 2: Practical functions of symbols.

Realizing parallel operation on computer clusters is a lowcost and high-efficiency solution.

Assuming that $f(m, n)$ is a function with two discrete spatial variables $m, n$, the definition of the two-dimensional Fourier transform of the function is as follows:

$$
F\left(\omega_{1}, \omega_{2}\right)=\sum_{m=-\infty}^{\infty} \sum_{n=-\infty}^{\infty} f(m, n) e^{-j \omega_{1} m} e^{-j \omega_{2} m},
$$

where $\omega_{1}, \omega_{2}$ are variables in the frequency domain, and its unit is radian/sampling unit. Usually, the function $F\left(\omega_{1}, \omega_{2}\right)$ is called the frequency domain representation of $f(m, n)$, and $F\left(\omega_{1}, \omega_{2}\right)$ is a complex function, and the period of its variables $\omega_{1}, \omega_{2}$ is $2 \pi$.

Inverse Fourier transform is defined as follows:

$f(m, n)=\frac{1}{4 \pi^{2}} \int_{-\pi}^{\pi} \int_{-\pi}^{\pi} F\left(\omega_{1}, \omega_{2}\right) e^{-j \omega_{1} m} e^{-j \omega_{2} m} \mathrm{~d} \omega_{1} \mathrm{~d} \omega_{2}$.

Simply put, this equation shows that $f(m, n)$ can be expressed as the sum of an infinite number of complex functions with different frequencies. While the contribution of different frequency points $\left(\omega_{1}, \omega_{2}\right)$ is determined by the amplitude $F\left(\omega_{1}, \omega_{2}\right)$.

With discrete Fourier transform [24], a fast algorithm, namely FFT (fast Fourier transform), can be used.

The design idea of FFT is to divide the original function into odd and even terms. By adding (subtracting) an odd and even term continuously, the desired result can be obtained.

Let the discrete function $f(m, n)$ be nonzero in the finite region $0 \leq m \leq M-1,0 \leq n \leq N-1$, then the relationship between the two-dimensional $M \times N$ and the inverse FFT is as follows:

$$
W_{N}^{p m}=\exp \left(-j \frac{2 \pi p m}{N}\right)
$$

Therefore, the processing speed can be improved to the greatest extent only when the matrix is a power of 2 when FFT is performed on the image, so the image size of the experiment is a power of 2 .
GA (genetic algorithm) shows good convergence and high efficiency in data design. The elements that makeup cartoon characters are simulated and trained by this algorithm, and finally, the cartoon character shapes are obtained, aiming at improving the efficiency of the cartoon art design.

If the total number of elements of a character in comic design is $N$, then the fitness of each of the $N$ elements is $f_{i}$, then the probability of the evolution of the $i$-th element is

$$
P=\frac{f_{i}}{\sum_{i=1}^{N} f_{i}},
$$

where $i=1,2, \cdots, N$.

Let the position of the character change to $\delta(H)$ from the initial time to the last time in a certain period of time. While the probability of crossover and change of GA is $P_{c}, P_{m}$, respectively, then the expected value of the next generation of the dynamic movement of characters is as follows:

$$
\begin{aligned}
E[m(H, t+1)] \geq & m(H, t) \cdot \frac{f(H, t)}{\bar{f}(t)} \\
& \cdot\left[1-P_{c} \frac{\delta(H)}{L-1}-O(H) P_{m}\right],
\end{aligned}
$$

where $O(H)$ is the dynamic motion order of the character, $L$ is the longest distance of character movement, $m(H, t)$ is the number of elements needed for the dynamic movement of characters in the next generation, and $f(H, t), \bar{f}(t)$ is the fitness and mean fitness of the elements needed by the next generation of characters [25].

In this paper, the variance of population fitness is used as the criterion of convergence. With the continuous evolution, the variance of the whole population becomes smaller and smaller. In the development stage, according to the difference of current convergence progress, the method of adaptive adjustment of jumping position is adopted to 
prevent it from falling into the local optimum. The adjustment strategy is as follows:

$$
\begin{aligned}
& P_{w}= \begin{cases}P_{w}+D_{i}, & \sigma_{\text {now }}<\sigma_{\text {set }}, \\
\phi \times\left(P_{w}+D_{i}\right), & \sigma_{\text {now }} \geq \sigma_{\text {set }},\end{cases} \\
& D_{i}=\operatorname{rand} \times\left(P_{b}-P_{w}\right),
\end{aligned}
$$

where $P_{w}$ represents the frog with the worst fitness in the population, $D_{i}$ stands for jumping distance, $\sigma_{\text {now }}$, $\sigma_{\text {set }}$ is the fitness variance of the current population and the critical point of variance used to distinguish the evolution stage of the current population, and $\phi$ represents the contraction factor for dynamically adjusting the jumping position.

This method finely perturbs the current population and gives it the ability to break into local optimum. The above two improved strategies greatly improve the convergence speed and optimization ability of the algorithm.

The design evolution process is shown in Figure 3. At the end of evolution, experts choose to save the excellent solution as the initial population for future evolution. Therefore, continue to find satisfactory numbers along the original design direction and shorten the design time.

\section{Results Analysis and Discussion}

Visual symbols naturally shoulder the responsibility of inheriting culture as one of the cultural carriers while absorbing the spirit of traditional culture. Chinese design should be based on local culture, tap the spiritual connotation of Chinese traditional culture, and integrate it into the innovative design of visual symbols while actively introducing international language. Break through the stylization of form and ideographic expression, the original visual form and values, the constant surpassing and renewing of oneself in form, and the creation of conditions for the establishment of a new society. Make it more vibrant by giving it a new visual experience. Freshness as a traditional symbol can interact with modern society and demonstrate the vitality and aesthetic value.

Figure 4 is the extraction of gray-level equalization processing time of six images, all of which are in TIF format. Based on $256 \times 256$ images, they are scaled into different pixel images of $32 \times 32$ to $1,024 \times 1,024$, respectively. Because the test time is dynamic, that is, the test values of each time are not necessarily exactly the same but tend to be a constant value, so here, 15 experimental tests are carried out on each image, and then the average processing time is calculated once, and the time unit is seconds.

From the data in Figure 4, it can be seen that there is a certain difference in the occupied space of the image. Sampling and quantization processing are two key factors that determine the closeness between the final digital image and the original image and are also related to the size of the data.

If the designer wants to complete the communication of the whole design well, the first condition is to see if he can grasp all kinds of relevant information in the early stage of

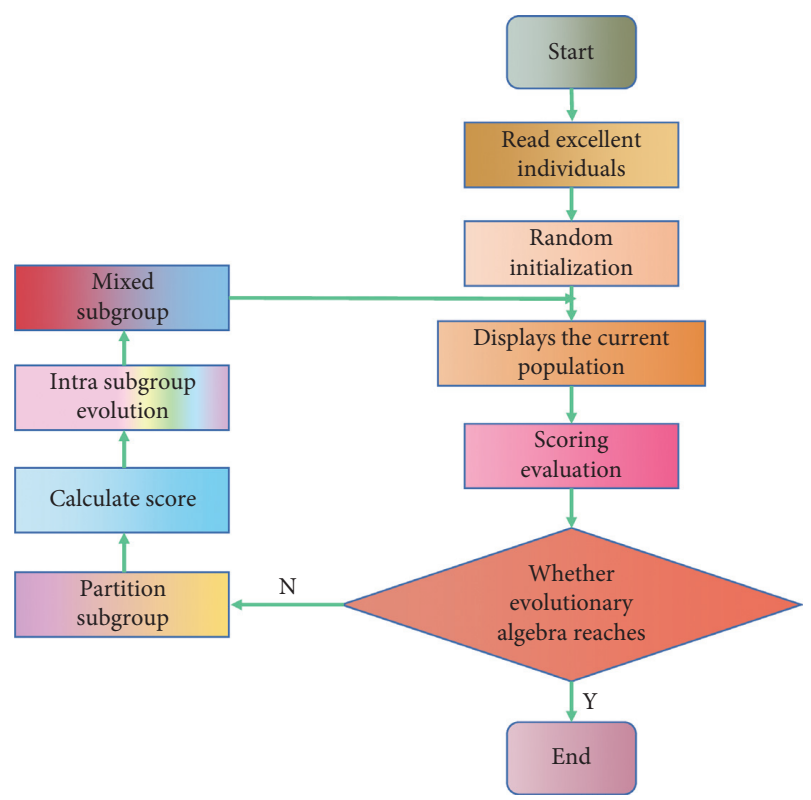

FigURe 3: Evolution process of animation design.

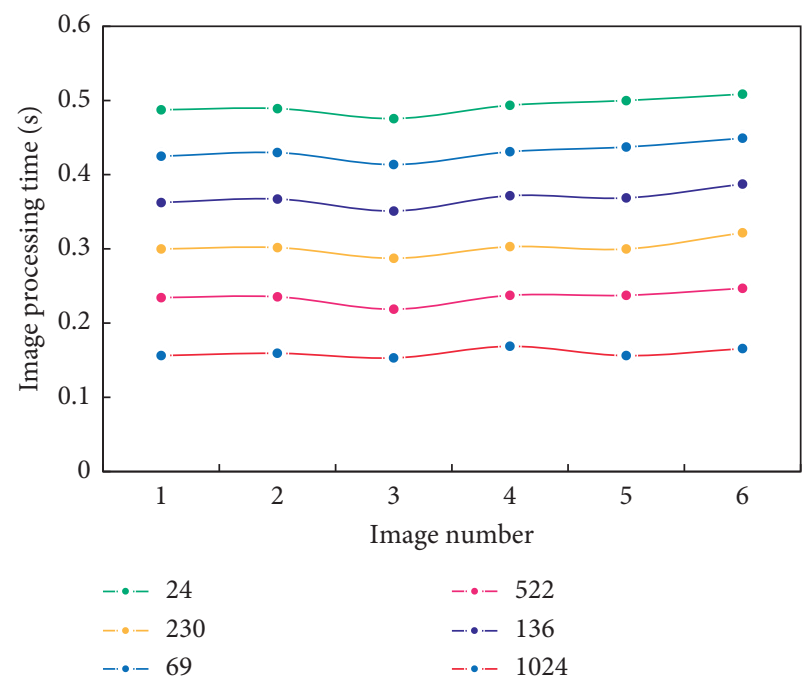

Figure 4: Experimental data.

the design communication process or whether he can dig out the driver of the consumer demand as the receiver or find the information points that the role modeling designer as the sender can use to form the message to achieve the expected effect.

Cinema animation belongs to the category of commercial animation, and its commercial operation mode is to produce animation according to market demand, including animation theme, animation picture, animation details, and so on. The freedom and randomness of character modeling design are completely inferior to experimental animation.

This price is not desirable when the time performance of the image is not very high. At the same time, it is unnecessary for this simple image processing method to adopt parallel 
processing. As the parallel processing of cluster computer system, it is aimed at the complex data processing with a large amount of data. Figure 5 is a time comparison curve of single- and multi-machine parallel processing.

From Figure 5, it is found that when the pixel value of the image is around 28 50, the time performance of single-machine processing is better than that of multimachine processing. Then, with the increase of pixel value, the time of single-machine processing increases rapidly, but the time of multi-machine parallel processing increases slowly. In other words, with the increase of image data, parallel processing shows its advantages, and the time of parallel processing is obviously lower than that of single-machine processing.

According to the character setting of roles and the ontological characteristics of basic elements, some relatively complex things are concentrated and summarized and modified by simplified methods. Because the animation character modeling is a nonreal character with strong exaggeration, when modeling, you need to deliberately strengthen the sense of rhythm. After the rhythm of the big shape is designed, the details are depicted. When adding details, do not usurp the host's role. Do not pay too much attention to details and ignore the comparison of the overall basic shape. Details should be refined, enriched, and perfected on the basis of the basic shape.

From the experimental results in Figure 6, it is found that the processing time of $32 \times 32$ and $64 \times 64$ pixel images is very close, and the processing time is also the shortest. If analyzed from the real-time point of view, they are most suitable for the requirements, but in the case of singlemachine processing, their resolution is low and the discrimination is poor.

From the processing time in Figure 6, it can also be found that the time spent on image sharpening and filtering accounts for a large part of the image processing time. Although their processing time is close when the pixels are small, it can still be verified that the complexity of this algorithm is relatively high.

In the experiment, it is also found that the edge image obtained by edge detection based on edge segmentation can meet the requirements of a certain environment, such as some requirements of infrared image processing. It does not have as much information as the gray image, but its information can describe the attributes of objects and has certain image information requirements. The biggest feature of this image is that it occupies very little storage space, as shown in Figure 7.

Using the Sobel operator to process the image edge detection has higher processing complexity than gray-level equalization and sharpening filtering, so the processing time is obviously increased. From the above experimental analysis of sharpening filtering, it can be seen that the Sobel operator is more suitable for parallel processing.

When creating works, the properties of the material should be respected. For example, to draw ethereal scene effects, choose watercolor materials such as watercolors, ink, and wash, which can create a sense of fluidity and create ethereal effects, or use relaxed lines to express them.

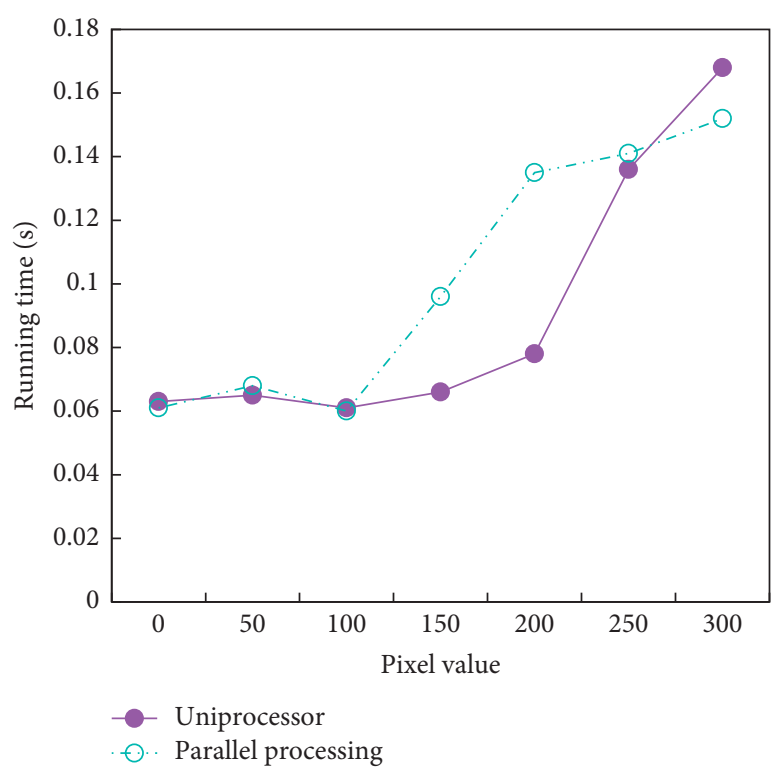

FIGURE 5: Comparison of single processor and parallel processing time.

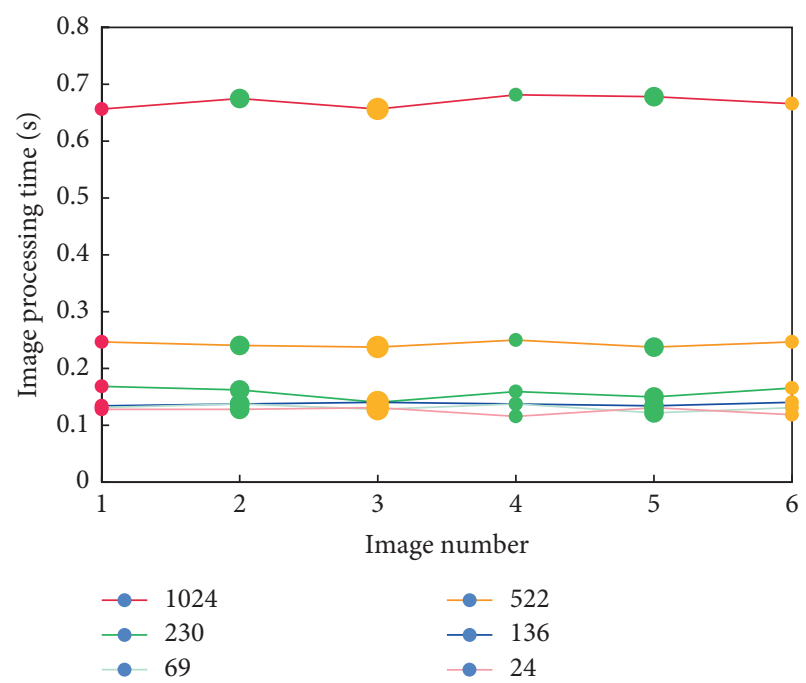

Figure 6: Time test of adaptive equalization Laplace filter.

This requires creators to face up to the materials and study and analyze the materials when creating, so as to obtain the correct psychological reflection of the materials and portray interesting works. The correct psychological response is also based on grasping the semantics of materials, respecting the attributes of materials, and maximizing the semantics of materials in the creative process. This is the basic criterion for the scientific and rational use of materials.

In order to test the effectiveness of the improved algorithm, this paper compares the algorithm in this paper, in reference [18], and in reference [23] with the classical test function, as shown in Figures 8 and 9.

Above all, because the algorithm in this paper dynamically and adaptively adjusts the jumping distance and 


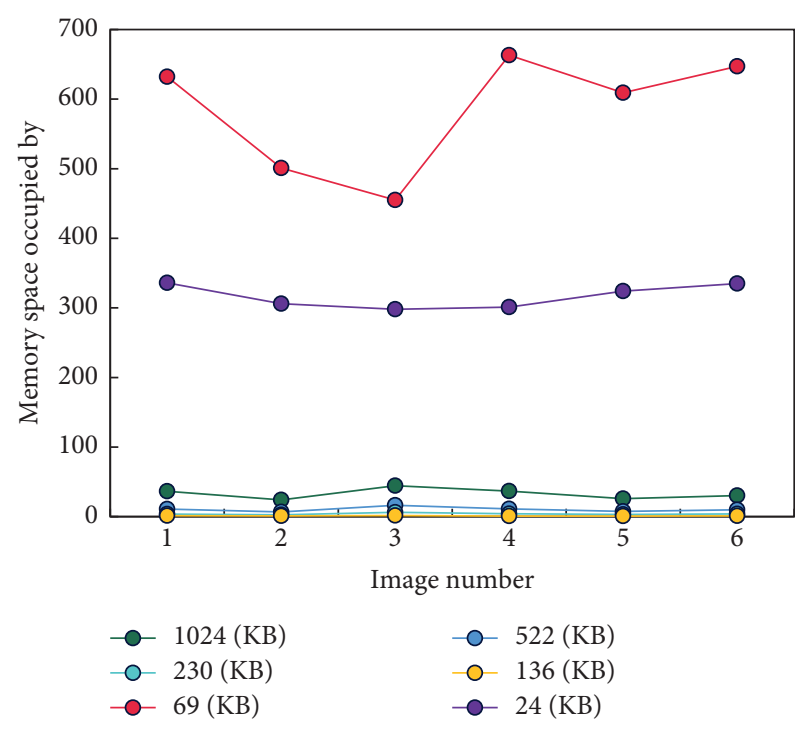

Figure 7: Memory space occupied by image after Sobel operator edge detection processing.

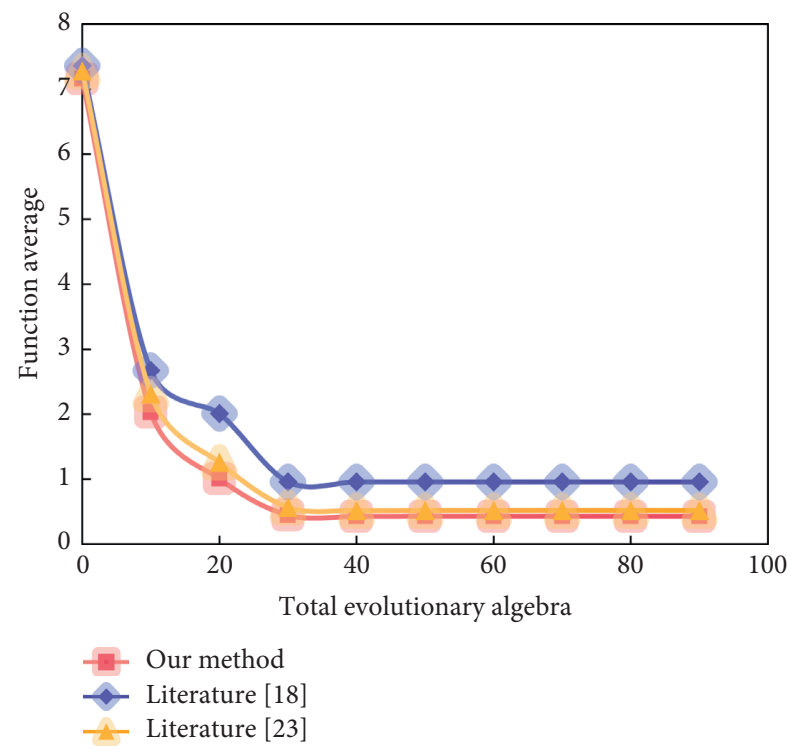

FIgURe 8: Ackley function test results.

disturbance position in the development stage, jumping out of the local extremum, and strengthening the global optimization ability of the algorithm, its optimization ability is stronger than that of literature [18] and [23].

When designing and creating, it is important to understand the basic emotional language of materials, as well as the innovative ways to use them. It is an important practical process of emotional experience for creators, regardless of which application it is. Through investigation, it was discovered that each material has its own semiotic meaning or symbolic semantics. The charting of the creative process clearly shows the creative intention, the beginning of a painting, the painting process, and the thinking in painting, which not only conveys the feeling that the creator wants to

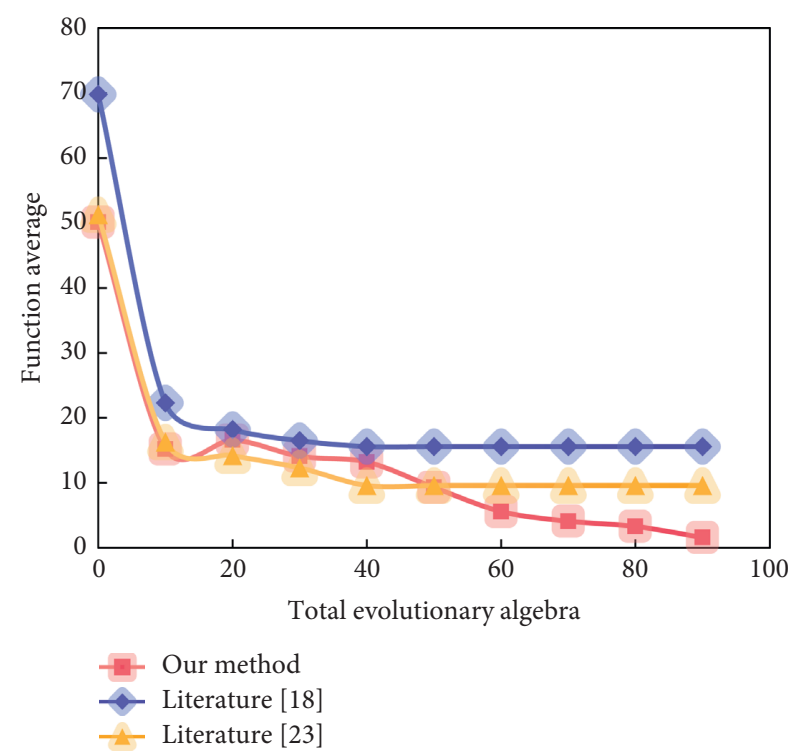

Figure 9: Griewank function test results.

express to the viewer but also serves as a kind of thinking reference, providing practical support and theoretical verification for other creators' creations.

Calculate the optimal solution of the dynamic change of character modeling obtained by the three algorithms, as shown in Figure 10.

It can be seen from Figure 10 that with the increase of GA evolutionary algebra, the fitness value decreases continuously. When the evolutionary algebra increases continuously, the fitness value finally decreases to a stable value, and the algorithm converges to obtain the optimal solution of the algorithm. In this paper, the algorithm falls into the minimum interval of local optimum, and the evolutionary algebra can change from local optimum to global optimum after only 7 times.

Symbolization of animation modeling is actually a process of combining abstract thinking activities in our minds through figurative things or symbols and creating new symbols by colonization. And this process is the process of modeling, but when it comes to modeling, it will inevitably involve the problem of shape. No successful animation character modeling can only be represented by a simple modeling element, but it is often dominated by a certain form element, which is compounded by multiple or multiple form elements. Therefore, it is necessary for animation character modeling design to understand the attribute characteristics of these three basic configuration elements. With the development of modern science and technology, the innovation of animation materials is inexhaustible, and the combination of various materials and animation forms will surely be a permanent proposition for animation creators and technicians. Taking materials as the starting point of animation creation can vividly reflect the change of people's ideas, the progress, and development of eulogizing society and provide more possibilities for animation art innovation. 


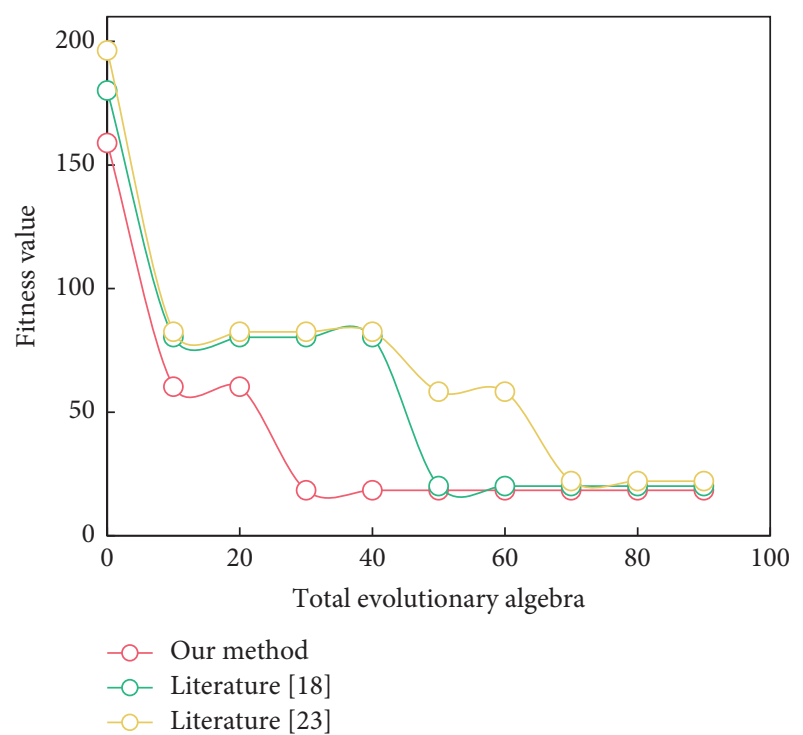

FIgURE 10: Optimization results of three algorithms.

\section{Conclusion}

New technical materials have a wide range of applications in cartoon character modeling, including clothing, clothing decoration structure, and props. The important direction of its technical development is to achieve a richer display effect through hardware structure, which is a key component of personalized and vivid clothing. New applications in the design of cartoon characters can be obtained when these new materials and technologies are combined. The modeling design in cinema animation is based on the script's basic information and values, which uses visual symbols to cut into the design and transforms them into visual symbols to express in the modeling, allowing viewers to understand the modeling expression language. The improved GA is used in this paper to display the dynamic changes in cartoon character modeling design, which can better complete the dynamic display of cartoon character modeling while also improving the algorithm's execution efficiency.

In the next step, machine learning-based automatic scoring will be used to reduce the time it takes to evaluate user interactions while also improving the action fidelity of cartoon characters in group sports.

\section{Data Availability}

The data used to support the findings of this study are available from the corresponding author upon request.

\section{Conflicts of Interest}

The author does not have any possible conflicts of interest.

\section{Acknowledgments}

This study was supported by The study School level scientific research projects by "Application of Shaanxi dough modeling arts plastic arts elements in animation modeling (Grant No. XJ200111).”

\section{References}

[1] J. Zhou, M. Campen, D. Zorin, C. Tu, and C. T. Silva, "Quadrangulation of non-rigid objects using deformation metrics," Computer Aided Geometric Design, vol. 62, pp. 3-15, 2018.

[2] P. Huber, R. Perl, and M. Rumpf, "Smooth interpolation of key frames in a Riemannian shell space," Computer Aided Geometric Design, vol. 52-53, pp. 313-328, 2017.

[3] S.-K. Wong, T.-C. Chang, T.-C. Ho, and J.-H. Chuang, "Fire synthesis using basis fires and design," The Visual Computer, vol. 33, no. 3, pp. 343-354, 2017.

[4] N. Kang, J. Bai, and J. Pan, "Interactive animation generation of virtual characters using single RGB-D camera," The Visual Computer, vol. 35, no. 6-8, pp. 849-860, 2019.

[5] S. Sebastian, H. Norman, and J. Zhang, "Memetic evolution for generic full-body inverse kinematics in robotics and animation," IEEE Transactions on Evolutionary Computation, vol. 23, no. 3, pp. 406-420, 2018.

[6] G. Albrecht, C. V. Beccari, J.-C. Canonne, and L. Romani, "Planar pythagorean-hodograph B-spline curves," Computer Aided Geometric Design, vol. 57, no. oct, pp. 57-77, 2017.

[7] M. Koehler, N. S. Usevitch, and A. M. Okamura, "Modelbased design of a soft 3-D haptic shape display," IEEE Transactions on Robotics, vol. 36, no. 3, pp. 613-628, 2020.

[8] H. Chen, H. Yang, and G. Yang, "3D product display based on inventor animation design," Chinese Journal of Scientific Instrument: English version, vol. 1, p. 9, 2020.

[9] Chen, Y. Hua, and Y. Hao, "3D product display based on inventor animation design," Journal of Instrumentation, vol. 7, no. 1, pp. 35-43, 2020.

[10] Y. Zhu, "Research on the application of Chinese folk art patterns in animation design," International technical management, vol. 2, p. 3, 2017.

[11] M.-Y. Song, E.-Y. Jo, and S.-H. Nam, "An authoring tool design for interactive animation contents based on android platform," Proceedings of the Korean Society of Computer Information Conference, vol. 25, no. 2, pp. 37-38, 2017.

[12] M. Brehmer, B. Lee, and P. Isenberg, "A comparative evaluation of animation and small multiples for trend visualization on mobile phones," IEEE Transactions on Visualization and Computer Graphics, vol. 26, no. 1, pp. 364-374, 2019.

[13] M. F. Stallmann, "Algorithm animation with galant," IEEE Computer Graphics and Applications, vol. 37, no. 1, pp. 8-14, 2017.

[14] J. Tan, "Evolution of art form of video animation design under the background of computer graphics system development," International Technical Management, vol. 6, p. 3, 2017.

[15] D. Li, H. Cao, S. Xi, X. Linand, and X. Chen, "Design optimization of motorized spindle bearing locations based on dynamic model and genetic algorithm," Transactions of the Canadian Society for Mechanical Engineering, vol. 41, no. 5, pp. 787-803, 2017.

[16] X.-q. Liu, Y. Jiang, F.-l. Liu, Z.-w. Liu, Y.-j. Chang, and G.-m. Chen, "Optimization design of fairings for VIV suppression based on data-driven models and genetic algorithm," China Ocean Engineering, vol. 35, no. 1, pp. 153-158, 2021.

[17] Y. Jiang, G. Tong, and H. Yin, "A pedestrian detection method based on genetic algorithm for optimize XGBoost training parameters," IEEE Access, vol. 99, p. 1, 2019. 
[18] M. Tarokh and H. D. Ho, "Kinematics-based simulation and animation of articulated rovers traversing uneven terrains," Robotica, vol. 37, no. 6, pp. 1057-1072, 2019.

[19] B. Matthew, R. D. Averett, and W. Xianqiao, "Flow plate separation of cells based on elastic properties: a computational study," Biomechanics and Modeling in Mechanobiology, vol. 18, no. 2, pp. 425-433, 2019.

[20] W. Xia, S. Han, N. Ullah et al., "Design and modeling of threedimensional laser imaging system based on streak tube," Applied Optics, vol. 56, no. 3, p. 487, 2017.

[21] S. A. Boltia, A. T. Soudi, and E. S. Elzanfaly, "Effect of genetic algorithm-based wavelength selection as a preprocessing tool on multivariate simultaneous determination of paracetamol, orphenadrine citrate, and caffeine in the presence of p-aminophenol impurity," Journal of AOAC International, vol. 1, p. 1, 2020.

[22] I. Changik, J. Kim, and N.-K. Cho, "Analysis of interface phenomena for high-performance dual-stacked oxide thin-film transistors via equivalent circuit modeling," ACS Applied Materials And Interfaces, vol. 13, no. 43, pp. 51266-51278, 2021.

[23] D. D. Li and X. Y. Liu, "Research on MVP design pattern modeling based on MDA," Procedia Computer Science, vol. 166, pp. 51-56, 2020.

[24] L. Mingxue, Y. Guolai, and L. Xiaoqing, "Variable universe fuzzy control of adjustable hydraulic torque converter based on multi-population genetic algorithm," IEEE Access, vol. 1, 2019.

[25] G. I. Henshall, W. J. Teahan, and L. A. CenyDd, "Virtual reality's effect on parameter optimisation for crowd-sourced procedural animation," Visual Computer, vol. 34, no. 9, 2018. 